\title{
Dynamical model for phase coexistence in proton glass
}

V. Hugo Schmidt

Citation: AIP Conference Proceedings 436, 192 (1998); doi: 10.1063/1.56272

View online: https://doi.org/10.1063/1.56272

View Table of Contents: http://aip.scitation.org/toc/apc/436/1

Published by the American Institute of Physics

\section{Conference Proceedings}

\section{Get $30 \%$ off all print proceedings!}

Enter Promotion Code PDF30 


\title{
Dynamical Model for Phase Coexistence in Proton Glass
}

\author{
V. Hugo Schmidt \\ Physics Department, Montana State University, Bozeman, MT 59717
}

\begin{abstract}
We describe a model for static and dynamic behavior of $\mathrm{KH}_{2} \mathrm{PO}_{4}(\mathrm{KDP})$ type crystals in the ferroelectric phase, and for proton glass crystals of this type in the temperature and composition range in which the ferroelectric and paraelectric phases coesist. Model predictions are compared with experimental results for ferroelectric $\mathrm{RbH}_{2} \mathrm{AsO}_{4}$ (RDA) and for the proton glass $\mathrm{Rb}_{1-x}\left(\mathrm{NH}_{4}\right)_{\mathrm{x}} \mathrm{H}_{2} \mathrm{AsO}_{4}$ (RADA). The model is based on the statistical mechanics and dynamics of a fundamental excitation in the ferroelectric phase, namely chains consisting of nonpolar $\mathrm{H}_{2} \mathrm{AsO}_{4}$ groups with an $\mathrm{H}_{3} \mathrm{AsO}_{4}$ group at one end and an $\mathrm{HAsO}_{4}$ group at the other end.
\end{abstract}

\section{INTRODUCTION}

Proton glasses offer a rich variety of phenomena, and are of particular interest because their dynamical processes can be understood in terms of simple excitations. We reported coexistence of ferroelectric and paraelectric phases in $\mathrm{Rb}_{1-\mathrm{x}}\left(\mathrm{NH}_{4}\right)_{\mathrm{X}} \mathrm{H}_{\mathrm{x}} \mathrm{AsO}_{4}$ (RADA) based on dielectric permittivity (1-3) and spontaneous polarization (3) measurements. We have employed other methods and studied other crystals as well, and have discussed the nature of such coexistence $(4,5)$. This paper develops a model which explains some of the phenomena observed in the coexistence region of the phase diagram.

\section{DESCRIPTION OF MODEL}

This model for static and dynamic properties of coexistence is based on the statistics of the most prevalent excitations in ferroelectric domains in KDP-type crystals. We choose $\mathrm{Rb}_{1-x}\left(\mathrm{NH}_{4}\right)_{\mathrm{x}} \mathrm{H}_{2} \mathrm{AsO}_{4}$ (RADA) as the crystal with which we compare model and experimental results in this work. In such crystals, these excitations are chains consisting of an $\mathrm{H}_{3} \mathrm{AsO}_{4}$ group and an $\mathrm{HAsO}_{4}$ group connected by nonpolar $\mathrm{H}_{2} \mathrm{AsO}_{4}$ groups. A nonpolar $\mathrm{H}_{2} \mathrm{AsO}_{4}$ group has energy $\varepsilon_{0}$, whereas a polar $\mathrm{H}_{2} \mathrm{AsO}_{4}$ group has zero energy, according to the Slater model (6). Each $\mathrm{AsO}_{4}$ arsenate ion is connected by four $\mathrm{O}-\mathrm{H} \cdots \mathrm{O}$ "acid" hydrogen bonds to neighboring arsenate ions. Most arsenate

\section{CP436, First-Principles Calculations for Ferroelectrics edited by Ronald $\mathrm{E}$. Cohen}

(C) 1998 The American Institute of Physics 1-56396-730-8/98/\$15.00 
ions have two protons close (hence the $\mathrm{H}_{2} \mathrm{AsO}_{4}$ designation for such groups), in these four bonds in which protons occupy off-center positions. If both bottom (relative to the $c$ axis) protons are close, the $\mathrm{As}^{5+}$ ion is pushed upward and the group has a positive electric dipole moment $\mu$ along $c$. If both top protons are close, the moment is negative. The remaining four $\mathrm{H}_{2} \mathrm{AsO}_{4}$ proton arrangements produce dipole moments in the $a b$ plane and contribute to dielectric permittivity in that plane. They are called nonpolar because they have no moment along $c$, the axis for ferroelectric polarization.

A chain of such nonpolar groups must terminate with a "Takagi" group of energy $\varepsilon_{1}$, typically about $5 \varepsilon_{0}$ in KDP-type crystals. Takagi (7) incorporated them into Slater's model and obtained better agreement with experiment. The reason for existence of such chains can be understood by following the dynamics of creation and growth of such a chain. Creation occurs within, say, a positive ferroelectric domain by means of one intrabond proton transfer,

$$
\mathrm{H}_{2} \mathrm{AsO}_{4}+\mathrm{H}_{2} \mathrm{AsO}_{4} \rightarrow \mathrm{H}_{3} \mathrm{AsO}_{4}+\mathrm{HAsO}_{4} .
$$

The proton is transferred to a site close to one of the two top oxygens of an $\mathrm{H}_{2} \mathrm{AsO}_{4}$ group, making it an $\mathrm{H}_{3} \mathrm{AsO}_{4}$ group and changing one of the two groups just above it to an $\mathrm{HAsO}_{4}$ group. This Takagi group pair has an a priori statistical weight of 2 , relative to 1 for the original polar ion at the bottom of this short chain. The chain can grow upward if a proton moves close to the top of the $\mathrm{HAsO}_{4}$ group, thereby converting it to a nonpolar $\mathrm{H}_{2} \mathrm{AsO}_{4}$ group because it has one close proton each at the top and bottom. The $\mathrm{HAsO}_{4}$ group has in effect moved to one of two possible positions higher along the $c$ axis, thereby again doubling the a priori statistical weight of the chain, to 4 . The chain energy has now increased from $2 \varepsilon_{1}$ to $2 \varepsilon_{1}+\varepsilon_{0}$. We ignore the possibility that the last proton moves away from the $\mathrm{HAsO}_{4}$ group, because that would entail a large energy increase, of order $\varepsilon_{1}$.

In general, if no ammonium ions are encountered, the Boltzmann factor $W_{n S}$ in the Slater limit for a chain of $\mathrm{n}$ links, involving $\mathrm{n}$ proton transfers, is

$$
W_{n S}=2^{n} \exp \left\{-\left[2 \varepsilon_{1}+(n-1) \varepsilon_{0}\right] / k T\right\} .
$$

For each step that the chain lengthens, the Boltzmann factor increases by the factor $2 \exp \left(-\varepsilon_{0} / \mathrm{kT}\right)$. We pointed out long ago (8) that the Slater model ferroelectric transition temperature

$$
T_{c}(\text { Slater })=\varepsilon_{0} / k \ln 2
$$

is exactly the temperature at which growth of the chain by one step does not change the Boltzmann factor, thereby allowing such chains to grow without limit and destroy the ferroelectric order.

This perfect agreement of the chain model with the Slater model hinges on being at the Slater limit, for which $\varepsilon_{1} \rightarrow \infty$. For finite Takagi energy $\varepsilon_{1}$, a large number of chains 
of finite length will destroy the ferroelectric order at a temperature below the Slater transition temperature.

\section{APPLICATION OF MODEL TO RDA}

As a first step in developing the chain model, without the complication of randomly placed ammonium ions, we apply it to pure $\mathrm{RbH}_{2} \mathrm{AsO}_{4}$ (RDA) and compare results with experiment and with Takagi model predictions. If $\mathrm{W}_{\mathrm{n}}$ is the probability that a chain of $\mathrm{n}$ links has its bottom end at a given arsenate anion site, and one notes that each link destroys the dipole moment of one arsenate anion (more exactly, of one formula unit), then the ferroelectric order parameter $\mathrm{p}$ is given by

$$
p=I-\Sigma n W_{n}=I-\Sigma\left(n W_{n S}\right) / \Sigma W_{n S}
$$

These sums, and all sums over $\mathrm{n}$ in this work, run from zero to infinity. The $\mathrm{W}_{\mathrm{ns}}$ for $\mathrm{n}=0$ is 1 , and the others are provided by Eq. (2). Upon evaluating these sums, we find

$$
\left.p(\operatorname{ch} a i n) \cdot(a-b c)(a+b c) /(a-b c)(a+b c)+b^{2} / 2\right]
$$

whereas the Takagi result is

$$
p(\text { Takagi })=[(a-b)(a+b)]^{1 / 2} / a
$$

Here, $a=1-2 \exp \left(-\varepsilon_{0} / k T\right), b=2 \exp \left(-\varepsilon_{1} / k T\right)$, and $c=\exp \left(-\varepsilon_{0} / 2 k T\right)$

The transition temperature $T_{c}$ is the lowest temperature at which $p$ becomes zero The transition temperatures for these models are given by

$$
\begin{aligned}
& T_{c}(\text { chain }) \rightarrow a-b c=1-2 \exp \left(-\varepsilon_{0} k T_{c}\right)-2 \exp \left\{-\left(\varepsilon_{I}+0.5 \varepsilon_{0}\right) / k T_{c}\right\}=0, \\
& T_{c}(\text { Takagi }) \rightarrow a-b=1-2 \exp \left(-\varepsilon_{0} k T_{c}\right)-2 \exp \left(-\varepsilon_{I} / k T_{c}\right)=0 .
\end{aligned}
$$

The chain model result for $p$ differs from the Takagi result in that $p$ (chain) has finite slope at $T_{c}$ (chain), while the Takagi model predicts infinite slope at $T_{c}$ (Takagi) as expected for a second-order transition. This defect of the chain model occurs because intersection of chains is neglected, and this neglect becomes serious near the transition. Neither model predicts the measured weakly first-order nature of the transition. Senko (9) added a long-range interaction to the Takagi model, which was shown by Silsbee, Uehling, and Schmidt (10) to change the transition to first order if this long-range interaction is large enough.

To make numerical and graphical comparison of these model predictions with experiment, we use the experimental value $T_{c}=109.75 \mathrm{~K}$ given by Fairall and Reese (11) for RDA, and the value $\varepsilon_{1} / \mathrm{kT}_{\mathrm{c}}=4.20$ provided by them, which corresponds to 
$\varepsilon_{1} / \mathrm{k}=460.95 \mathrm{~K}$. Because they included both the above-mentioned long-range interaction, and the tunneling effect introduced by Blinc and Svetina (12), their value for $\varepsilon_{0} / k T_{c}$ is much too low to provide the measured $T_{c}$ if only it and their $\varepsilon_{1} / k T_{c}$ are used in the Takagi model. Accordingly, we instead use for $\varepsilon_{0} / \mathrm{k}$ the value $79.41 \mathrm{~K}$ which when used in the Takagi model together with $\varepsilon_{1} / \mathrm{k}=460.95 \mathrm{~K}$ gives the measured $T_{c}$. With these values for $\varepsilon_{0}$ and $\varepsilon_{1}$, which force the Takagi result for $T_{c}$ to coincide with the experimental value, we obtain for $T_{c}$ given in Eqs. (3), (7), and (8) for the various models the values

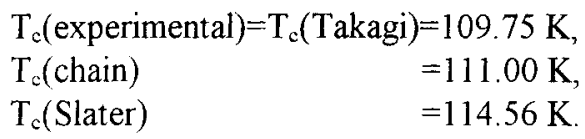

\section{APPLICATION OF MODEL TO RADA}

The reasonable agreement of the chain model result with the Takagi result encourages us to apply an extension of this chain model to proton glass crystals in the range of $\mathrm{x}$ for which coexistence of ferroelectric and paraelectric/proton glass phases occurs. This extension provides for a reduction in the chain energy wherever the chain passes by an ammonium ion. Although each cation site has probability $\mathrm{x}$ of containing an ammonium instead of a rubidium ion, this is not a mean field model because we specifically take into account the preference for chains to choose paths with neighboring ammonium sites. Taking this preference into account yields the non-meanfield result that even at zero temperature, the crystal contains both ferroelectric and proton glass phase regions for all $\mathrm{x}$ between 0 and $\mathrm{x}_{\mathfrak{c}}$, the concentration at which ferroelectricity disappears at zero temperature.

To take the effect of ammonium ions into account, we use an interaction we introduced previously (13) in an extension of the Slater model. This is the "crosscation interaction" which lowers the energy by $\varepsilon_{\mathrm{a}}$ whenever the two "acid" hydrogens opposite each other across an ammonium ion occupy sites consistent with an antiferroelectric rather than a ferroelectric configuration. There are two such hydrogen pairs for each ammonium ion, so the potential for the ammonium ion to destroy ferroelectric order is not exhausted until two acid hydrogens adjacent to the ammonium ion have moved away from their ordered-phase positions. Each acid hydrogen is bonded to two oxygens, and each of these oxygens can form an $\mathrm{N}-\mathrm{H} \ldots \mathrm{O}$ bond to a different ammonium ion if such an ion is in that cation site. If the chain has $\mathrm{n}$ displaced hydrogens and runs past $\mathrm{m}$ ammonium ions, its unnormalized Boltzmann factor $\mathrm{W}_{\mathrm{nu}}$, the mixed-crystal analog of $W_{\mathrm{nS}}$ in Eq. (2), is

$$
W_{n m u}=\left[2^{n}(1-y)^{2 n-m} y^{m}(2 n) ! /(2 n-m) ! m !\right] f\left(U_{n m}\right) \text {. }
$$


Here $\mathrm{y}$ is the effective $\mathrm{x}$, taking into account the above-mentioned saturation of the tendency of ammonium ions to destroy ferroelectric order, and given by

$$
y=x-0.5 \Sigma\left(m W_{n m u}\right) / \Sigma W_{n m u}
$$

Here and in the following equations, $\Sigma$ signifies a sum over $n$ from zero to infinity, and a sum over $m$ from zero to $2 \mathrm{n}$. After solving Eqs. (9) and (10) self-consistently for y, the ferroelectric order parameter is found from the analog of Eq. (4), namely

$$
p=1-\Sigma\left(n W_{n m}\right)=1-\Sigma\left(n W_{n m u}\right) / \Sigma W_{n m u}
$$

The factor $f\left(U_{n m}\right)$ in Eq. (9) depends on the chain energy $U_{n m}$, given by

$$
U_{n m}=2 \varepsilon_{l}+(n-1) \varepsilon_{l}-m \varepsilon_{\alpha}
$$

One might think that $f\left(U_{n m}\right)$ should have the Boltzmann form seen in Eq. (2), but use of that form in this calculational method would give completely erroneous results for mixed crystals. The reason for such erroneous results is that sufficiently large $\mathrm{m}$ can give negative $U_{n m}$. If we would construct a finite-size crystal with a given ammonium ion distribution, the ground state corresponding to zero temperature would contain a number of such chains with negative energies of various magnitudes. Instead, we are looking at all the possible chains that can begin at one given anion site, in a crystal of infinite extent. If we would employ Boltzmann statistics, then at zero temperature only the lowest energy state (of energy negative infinity!) would be occupied. A better choice for $\mathrm{f}\left(\mathrm{U}_{\mathrm{nm}}\right)$ would be the Fermi-Dirac distribution function, even though we are dealing with distinguishable particles so that there is no physical necessity for using Fermi-Dirac statistics. A simpler choice which also avoids the above low-temperature catastrophe would be to choose

$$
f=1 \text { for } U_{n m} \leq 0, f=\exp \left(-U_{n m} / k T\right) \text { for } U_{n m} \geq 0
$$

The $f=1$ choice is consistent with the unnormalized probability $W_{00 u}=1$ which must be used in the above sums, and simply means that no given configuration of a chain beginning at a single site can have more than single occupancy.

The possibility of negative $U_{n m}$ in Eq. (12) justifies the above statement that a mixed crystal cannot be in a completely ordered ferroelectric state. If in Eq. (12) we would replace $m$ by its mean-field $(m-f)$ value $2 n x$, then at zero temperature the crystal would have complete ferroelectric order for $\mathrm{x}<\mathrm{x}_{\mathrm{cm}-\mathrm{f}}$, and would be completely in the proton glass state for larger $x$ values below the antiferroelectric order range. This mean-field critical concentration is given by

$$
x_{c m-f}=\varepsilon_{v} / 2 \varepsilon_{a}
$$


This mean-field approximation seriously overestimates $x_{c}$. To find the degree of overestimation, and to obtain results from the chain model for mixed crystals, we need a numerical value for the cross-cation interaction energy $\varepsilon_{\mathrm{a}}$. This is obtained from our Slater-type model (13) for proton glass crystals which incorporates this interaction. For the large $\mathrm{x}$ values for which an antiferroelectric transition occurs, that model predicts a first-order transition with a jump from 0 to 1 for the antiferroelectric order parameter. This all-or-nothing jump is a consequence of setting the Takagi interaction energy $\varepsilon_{1}$ to infinity in that model. Such a jump occurs also for the ferroelectric order parameter $\mathrm{p}$ predicted by that model for lower $\mathrm{x}$, except in the Slater model limit $(\mathrm{x}=0)$, where all values $0 \leq \mathrm{p} \leq 1$ are allowed. (One can say that the Slater transition is locked to a tricritical point.) The actual transition in $\mathrm{NH}_{4} \mathrm{H}_{2} \mathrm{AsO}_{4}(\mathrm{ADA})$ is strongly first-order and occurs at $216.1 \mathrm{~K}$. Our Slater-type model predicted that this transition occurs for $\mathrm{ADA}$ at the "Néel" temperature $\mathrm{T}_{\mathrm{N}}$ for which the paraelectric and antiferroelectric free energies are equal. The condition for this equality is

$$
\varepsilon_{a}=f_{N} \varepsilon_{1 /}-k T_{N}\left[f_{N} \ln \left(2 f_{N}\right)+\left(1-f_{N}\right) \ln \left(1-f_{N}\right)\right],
$$

where $\mathrm{f}_{\mathrm{N}}$ is the fraction of zero-energy polar Slater groups in the paraelectric phase at temperature $T_{N}$, given by

$$
f_{N}\left(\text { paraelectric, } T_{N}\right)=\left[1+2 \exp \left(-\varepsilon_{0}\left(k T_{N}\right)\right]^{-1}=\left[1+2 \exp \left(-T_{c} \ln 2 / T_{N}\right)\right]^{-1} .\right.
$$

Here we substitute the Slater model expression $T_{\mathrm{c}} \ln 2$ for $\varepsilon_{0} / \mathrm{k}$, and use the measured $T_{c}$ of $109.75 \mathrm{~K}$ for RDA. These values, together with $\mathrm{T}_{\mathrm{N}}=216.1 \mathrm{~K}$, yield $\varepsilon_{\mathrm{a}} / \mathrm{k}=116.06 \mathrm{~K}$ when inserted into Eq. (15) and (16). This value for $\varepsilon_{a} / k$, together with the value $T_{d} \ln 2$ for $\varepsilon_{0} / \mathrm{k}$, yield a mean-field critical concentration of $\mathrm{x}_{\mathrm{cm}-\mathrm{f}}=0.328$, whereas the experimental value for which ferroelectric behavior disappears even at zero temperature is near $\mathrm{x}_{\mathrm{c}}=0.16$.

This large discrepancy shows that mean-field models fail badly when applied to twophase coexistence situations in which the coexistence is partially spatial because of quenched cation disorder. These models give fairly accurate results in the paraelectric phase of RDA (Slater model) and the paraelectric phase of RADA and ADA (Slater model with cross-cation interaction added), and in the ferroelectric phase of a pure (not mixed) crystal such as RDA (Takagi model) for which there is no quenched cation disorder and consequently the coexistence of ferroelectric and paraelectric phases found at nonzero temperature is temporal rather than spatial.

Predictions of this chain model for the ferroelectric order parameter $p$, compared with experimental results derived from permittivity and spontaneous polarization measurements (1-3), are presented in Fig. 1 for RADA crystals with various fractional ammonium ion concentrations $x$. The predictions employ Eqs. (9-13) and the three energy parameters $\varepsilon_{0} / \mathrm{k}=79.41 \mathrm{~K}, \varepsilon_{1} / \mathrm{k}=460.95 \mathrm{~K}$, and $\varepsilon_{\mathrm{a}} / \mathrm{k}=116.06 \mathrm{~K}$ which were defined and provided numerical values as explained above. 


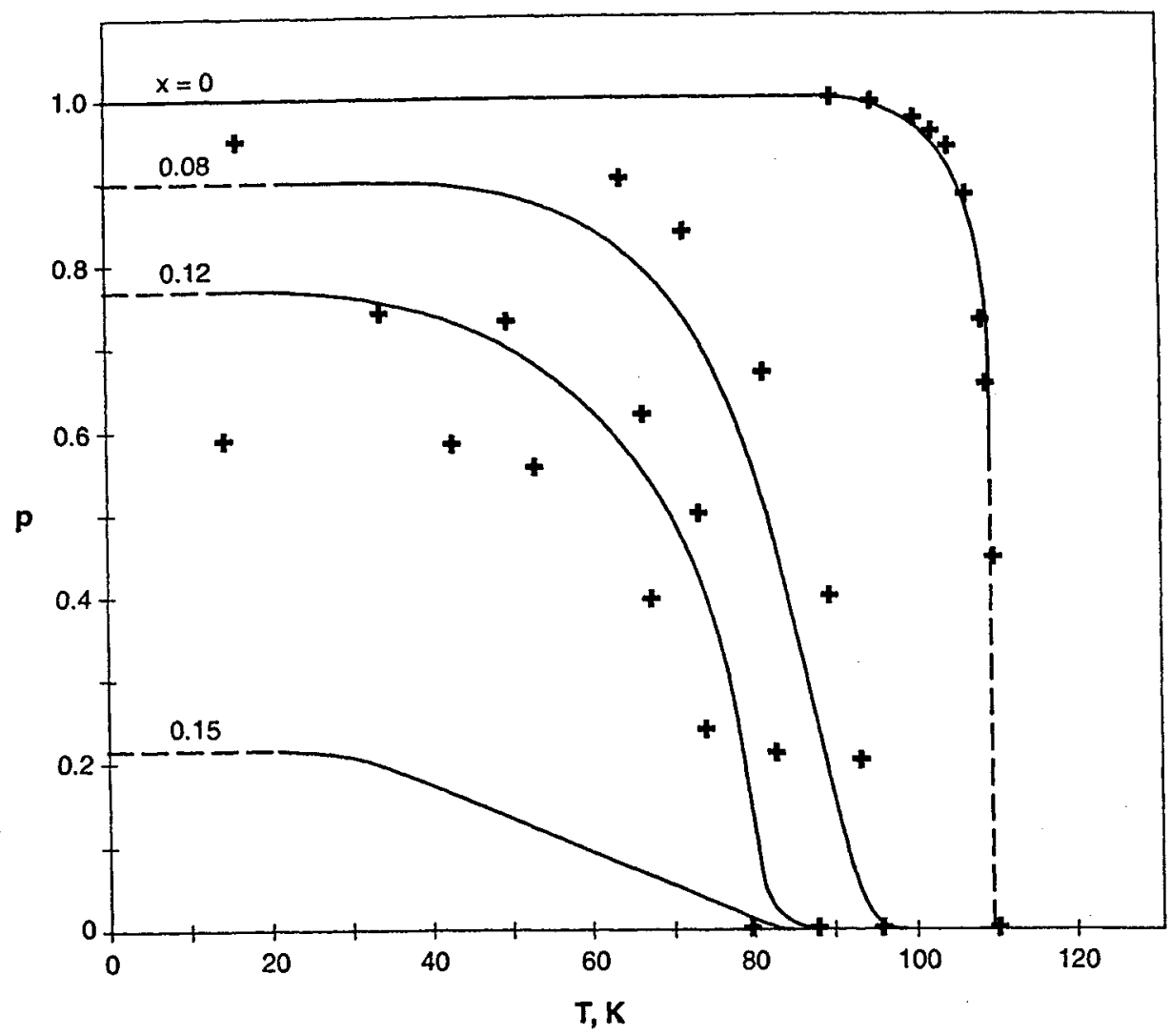

FIGURE 1. Comparison of temperature dependence of ferroelectric order parameter $p$ determined experimentally (solid and dashed lines) from permittivity and spontaneous polarization results by the method outlined in Ref. 3, for $\mathrm{Rb}_{1 \times x}\left(\mathrm{NH}_{4}\right)_{x} \mathrm{H}_{2} \mathrm{AsO} \mathrm{O}_{4}$ (RADA) crystals with $\mathrm{x}=0, \mathrm{x}=0.12$, and $\mathrm{x}=0.15$ (Ref. 1), and $x=0.08$ (Ref. 3), compared with predictions of the chain model (+) using Eqs. (9-13) as explained in the text.

The model fit shows promisingly good agreement with the experimental results. The $\mathrm{x}=0$ fit is excellent, the $\mathrm{x}=0.08$ calculated points are somewhat high, the $\mathrm{x}=0.12 \mathrm{fit}$ is better, and the $x=0.15$ calculated points are far too high. The qualitative features are nearly all correct; the order parameter $p$ saturates for low temperature at a level below 1 for nonzero $x$, the sharpness of curvature and the slopes of the straight portions are nearly right except for $\mathrm{x}=0.15$, and the temperatures at which $\mathrm{p}$ goes to zero are nearly correct. Only the small reverse bend seen for $\mathrm{x}=0.12$ and 0.08 near $\mathrm{p}=0$ is not predicted. The discrepancies are due to the model and perhaps also to experiment. The model is based on paraelectric chains, and loses accuracy for low $\mathrm{p}$ where the paraelectric region is quite extensive. The flame atomic-absorption spectroscopy used to determine $\mathrm{x}$ may have been inaccurate. Model refinements and additional fits to experiment will be made. 


\section{DIELECTRIC PERMITTIVITY AND RELAXATION}

The method for finding the dielectric permittivity and the dielectric response time constant is now illustrated, using RDA as an example. The contribution of the chains to the permittivity $\varepsilon$ is

$$
\varepsilon=\Delta P / \varepsilon_{M} E=\left(P_{s} / \varepsilon_{M}\right) d p / d E,
$$

where $\Delta \mathrm{P}$ is the polarization change, $\varepsilon_{\mathrm{M}}$ is the MKS constant $8.85 \times 10^{-12} \mathrm{C}^{2} / \mathrm{Nm}^{2}, \mathrm{E}$ is electric field applied along $c$, and $P_{\mathrm{s}}$ is the spontaneous polarization at zero temperature, $0.036 \mathrm{C} / \mathrm{m}^{2}$ for $\mathrm{RDA}$. We simplify the calculation without seriously affecting the result by using the Slater limit expression for $p$ in an electric field $E$, which is

$$
p=I-\exp \left[-\left(2 \varepsilon_{1}-\varepsilon_{0}\right) \cdot k T\right] \Sigma\left\{n 2^{n} \exp \left[-n\left(\varepsilon_{0}+\mu E\right) / k T\right]\right\},
$$

where $\mu=\mathrm{P}_{\mathrm{s}} \mathrm{a}^{2} \mathrm{c} / 4$ is the electric dipole moment per polar molecular unit, and a and $\mathrm{c}$ are the unit cell dimensions, both near $7.5 \times 10^{-10} \mathrm{~m}$, so $\mu$ is near $3.8 \times 10^{-30} \mathrm{C}-\mathrm{m}$. We see that $\mathrm{dp} / \mathrm{dE}$ is given by

$$
\begin{aligned}
& d p / d E=-\exp \left[-\left(2 \varepsilon_{I}-\varepsilon_{0}\right) / k T\right] \mu d \Sigma\left\{n 2^{n} \exp \left(-n \varepsilon_{0} k T\right)\right\} / d \varepsilon_{0} \\
& =-\exp \left[-\left(2 \varepsilon_{l}-\varepsilon_{0}\right) / k T\right] \mu d\left\{2 \exp \left(-\varepsilon_{0} / k T\right) /\left[1-2 \exp \left(-\varepsilon_{\theta} / k T\right)\right]^{2}\right\} / d \varepsilon_{0} .
\end{aligned}
$$

Thus the permittivity contribution of the chains is given by

$$
\varepsilon=\left(4 P_{s} \mu / \varepsilon_{M} k T\right) \exp \left(-2 \varepsilon_{l} k T\right)\left[1+2 \exp \left(-\varepsilon_{0} k T\right)\right] /\left[1-2 \exp \left(-\varepsilon_{0} k T\right)\right]^{3} .
$$

This ignores any contribution of domain wall mobility. The permittivity appears to be heading for a singularity, but because $\varepsilon_{1}$ is not infinite, the transition occurs before the singularity is reached.

The dielectric relaxation time constant $\tau$ is the final change in polarization $P$ (which is proportional to the order parameter $p$ ) induced by a small field step $E$, divided by the initial rate of change caused by that step,

$$
\tau=\Delta p /(d p / d t)=E(d p / d E) /(d p / d t)=\left(\varepsilon_{M} \varepsilon E / P_{s}\right) /(d p / d t),
$$

where $\varepsilon$ is the permittivity given in Eq. (20). To find dp/dt we use the common kinetic theory approximation that the jump rate $v$, for protons in this case, is

$$
v=v_{0} \exp (-\Delta U / k T) \text { if } \Delta U \geq 0, v=v_{0} \text { if } \Delta U \leq 0 \text {. }
$$


Here $\Delta \mathrm{U}$ is the energy change, including the field-induced change, resulting from the jump. We choose $v_{0}=k T / h$, where $h$ is Planck's constant, which is applicable because we are below the Debye temperature. Because $\mathrm{E}$ is small, the exponential in Eq. (22) can be expanded as

$$
\exp (-\Delta U / k T) \simeq \exp \left(-\Delta U_{0} k T\right)(1-\mu E / k T)
$$

for jumps which create or lengthen chains, which require positive energy $\Delta \mathrm{U}_{0}$ in the absence of a field, and which require additional energy $\mu \mathrm{E}$ in a field $\mathrm{E}$ along the ferroelectric polarization direction because the jump destroys a dipole moment of magnitude $\mu$. The field-induced rate of change of $p$ attributable to the Takagi group at the top of a chain is thus

$$
(d p / d t)_{1:}=2 W_{n S} v_{0} \exp \left(-\Delta U_{0} k T\right) \mu E / k T .
$$

A factor of 2 occurs because either of two protons can move in to that group and thus lengthen the chain. The $W_{n s}$ factor from Eq. (2) is the probability in the Slater limit that a chain of length $n$ begins at a given anion site. We take into account the fact that the given anion site, which is occupied either by a polar Slater group or by the bottom of a chain, is representative of the whole crystal, so one chain lengthening step reduces $\mathrm{p}$ by 1 . The energy change $\Delta \mathrm{U}_{0}$ is $\varepsilon_{0}$, except for the creation process, in which case it is $2 \varepsilon_{1}$. Summing the contributions from Eq. (3), keeping in mind that $W_{0 s}=1$, yields

$$
d p^{\prime} d t=(2 \mu E / h) \exp \left(-2 \varepsilon_{l} / k T\right) /\left[1-2 \exp \left(-\varepsilon_{0} / k T\right)\right] .
$$

Combining Eqs. (20), (21) and (25) yields

$$
\tau=(2 h / k T)\left[1+2 \exp \left(-\varepsilon_{0} k T\right)\right] /\left[1-2 x p\left(-\varepsilon_{0} k T\right)\right]^{2} .
$$

We find that $\tau$ decreases with decreasing temperature. This behavior of $\tau$ contradicts the common belief that time constant increases (because motion "freezes out") as temperature decreases in the ferroelectric phase. What "freezes out" is the amplitude of the dielectric response $\varepsilon$, as seen in Eq. (20). As with $\varepsilon$, $\tau$ does not become infinite with increasing temperature because the noninfinite Takagi energy $\varepsilon_{1}$ causes $T_{c}$ to occur before the denominator in Eq. (26) vanishes.

If we insert our previously determined values, $\varepsilon_{0} / \mathrm{k}=79.41 \mathrm{~K}$ and $\varepsilon_{1} / \mathrm{k}=460.95 \mathrm{~K}$, into Eq. (26), we obtain at $T_{c}=109.75 \mathrm{~K}$ the time constant $\tau\left(T_{c}\right)=1.92 \times 10^{-9} \mathrm{~s}$, which is much longer than the attempt time $\tau_{0}\left(T_{c}\right)=h / k T_{c}=4.37 \times 10^{-13} \mathrm{~s}$. Again using these values and other parameters listed above, Eq. (20) for permittivity $\varepsilon$ at $T_{c}$ the value $\varepsilon\left(T_{c}\right)=673$. This large value can be swamped by the domain wall contribution. 
Similar methods can be employed to find $\varepsilon$ and $\tau$ for the $a$ axis, and for both the $a$ and $c$ axes in the coexistence region for $0<\mathrm{x}<\mathrm{x}_{\mathrm{c}}$. A problem for the $a$ axis calculation is finding $\mu_{\mathrm{a}}$, because there is no phase ordered ferroelectrically along $a$, so the method used to find $\mu$ along $c$ from $\mathrm{P}_{\mathrm{s}}$ along $c$ has no analog for finding $\mu_{\mathrm{a}}$. The best approach may be to assume that the $\mu$ 's are proportional to the Curie-Weiss constants found from measurements in the paraelectric phase.

\section{CONCLUSIONS}

Most of the features of coexistence of the ferroelectric phase with the paraelectric/proton glass phase in $\mathrm{Rb}_{1-x}\left(\mathrm{NH}_{4}\right)_{\mathrm{x}} \mathrm{H}_{2} \mathrm{AsO}_{4}$ ( $\mathrm{RADA}$ ) proton glass crystals are predicted by a model which employs the statistics of chainlike excitations from the ferroelectric phase. Improvements to the model are needed particularly in the region of lower ferroelectric order parameter $p$. Additional fits to other dielectric data, and neutron diffraction and nuclear magnetic results, are needed for RADA and other proton and deuteron glasses, also in the region of coexistence of the antiferroelectric phase with the paraelectric/proton glass phase.

\section{ACKNOWLEDGEMENTS}

This work was supported by National Science Foundation Grant DMR-9520251. Thanks are expressed to George Tuthill for a helpful discussion.

\section{REFERENCES}

1. Trybula, Z., Schmidt, V. H., and Drumheller, J. E., Phys. Rev. B 43, 1287-1289 (1991).

2. Howell, F. L., Pinto, N. J., and Schmidt, V. H., Phys. Rev. B 46, 13762-13766 (1992).

3. Pinto, N. J., and Schmidt, V. H., Ferroelectrics 141, 207-213 (1993).

4. Schmidt, V. H., Trybula, Z., Pinto, N. J., and Shapiro, S. M., Phase Transitions (Proc. XVII Crystallography Congress, in press).

5. Schmidt, V. H., J. Korean Phys. Soc. (Proc. 9th Internat. Mtg. on Ferroelectricity, in press).

6. Slater, J. C., J. Chem. Phys. 9, 16-33 (1941).

7. Takagi, Y., J. Phys. Soc. Jpn. 3, 273-274 (1948).

8. Schmidt, V. H., Deuteron Jumping in $\mathrm{KH}_{2} \mathrm{PO}_{4}$, Ph.D. Thesis, University of Washington, 1961, pp. $90-91$

9. Senko, M. E., Phys. Rev. 121, 1599-1604 (1961).

10. Silsbee, H. B., Uehling, E. A., and Schmidt, V. H., Phys. Rev. 133, Al65-A170 (1964).

11. Fairall, C. W., and Reese, W., Phys. Rev. B 11, 2066-2068 (1975).

12. Blinc, R., and Svetina, S., Phys. Rev. 147, 423-429 and 430-438 (1966).

13. Schmidt, V. H., Wang, J. T., and Schnackenberg, P., Jpn. J. Appl. Phys. 24, Suppl. 24-2, 944-946 (1985); see corrections in Schmidt, V. H., J. Phys. Soc. Jpn. 56, 3752-3753 (1987). 